\title{
MARKOFF-ROSENBERGER TRIPLES WITH FIBONACCI COMPONENTS
}

\author{
SzabolCs TENGELY \\ University of Debrecen, Hungary
}

\begin{abstract}
We characterize the solutions of the Markoff-Rosenberger equation

$$
a x^{2}+b y^{2}+c z^{2}=d x y z
$$

with $a, b, c, d \in \mathbb{Z}, \operatorname{gcd}(a, b)=\operatorname{gcd}(a, c)=\operatorname{gcd}(b, c)=1$ and $a, b, c \mid d$, for which $(x, y, z)=\left(F_{i}, F_{j}, F_{k}\right)$, where $F_{n}$ denotes the $n$-th Fibonacci number for any integer $n \geq 0$.
\end{abstract}

\section{INTRODUCTION}

Markoff ([6]) obtained many nice results related to the equation

$$
x^{2}+y^{2}+z^{2}=3 x y z .
$$

He showed that there exist infinitely many integral solutions. The so-called Markoff equation defined above has been generalized in many directions by several authors. In this article we focus on the generalization considered by Rosenberger ([7])

$$
a x^{2}+b y^{2}+c z^{2}=d x y z .
$$

Rosenberger proved that if $a, b, c, d \in \mathbb{N}$ are integers such that $\operatorname{gcd}(a, b)=$ $\operatorname{gcd}(a, c)=\operatorname{gcd}(b, c)=1$ and $a, b, c \mid d$, then non-trivial solutions exist only if $(a, b, c, d) \in\{(1,1,1,1),(1,1,1,3),(1,1,2,2),(1,1,2,4),(1,1,5,5),(1,2,3,6)\}$. Silverman ([8]) studied equation (1.1) with $a=b=c=1$ over imaginary quadratic number fields. Baer and Rosenberger ([1]) considered solutions of equation (1.1) over imaginary quadratic number fields. González-Jiménez and Tornero ([4]) looked for solutions of equation (1.1) in arithmetic progression

2020 Mathematics Subject Classification. 11D45, 11B39.

Key words and phrases. Fibonacci numbers, Markoff equation. 
that lie in the ring of integers of a number field. González-Jiménez ([3]) studied solutions of (1.1) whose coordinates belong to the ring of integers of a number field and form a geometric progression. A well-known identity related to the Fibonacci numbers

$$
1+F_{2 n-1}^{2}+F_{2 n+1}^{2}=3 F_{2 n-1} F_{2 n+1}
$$

shows that $(x, y, z)=\left(1, F_{2 n-1}, F_{2 n+1}\right)$ is a solution of the Markoff equation for any $n \in \mathbb{N}$. Luca and Srinivasan ([5]) proved that there are infinitely many solutions $\left(F_{i}, F_{j}, F_{k}\right)$ to the classical Markoff equations (given by the above identity). In this paper we extend the result of Luca and Srinivasan, we determine the solutions $(x, y, z)=\left(F_{i}, F_{j}, F_{k}\right)$ of equation (1.1) for

$$
(a, b, c, d) \in\{(1,1,1,1),(1,1,2,2),(1,1,2,4),(1,1,5,5),(1,2,3,6)\} .
$$

In the proofs, we simplify the strategy described by Luca and Srinivasan, by providing a direct way to get a bound for $k-j$ from above.

\section{MAIN RESUlT}

THEOREM 1. If $(x, y, z)=\left(F_{i}, F_{j}, F_{k}\right)$ is a solution of equation (1.1) and $(a, b, c, d) \in\{(1,1,1,1),(1,1,2,2),(1,1,2,4),(1,1,5,5),(1,2,3,6)\}$, then the complete list of solutions are given by

\begin{tabular}{|c|c|}
\hline$(a, b, c, d)$ & solutions \\
\hline$(1,1,1,1)$ & $\{(3,3,3)\}$ \\
\hline$(1,1,2,2)$ & $\{(2,2,2)\}$ \\
\hline$(1,1,2,4)$ & $\{(1,1,1),(1,3,1),(1,3,5),(3,1,1),(3,1,5)\}$ \\
\hline$(1,1,5,5)$ & $\{(1,2,1),(1,3,1),(1,3,2),(2,1,1),(3,1,1),(3,1,2)\}$ \\
\hline$(1,2,3,6)$ & $\{(1,1,1),(1,2,1),(1,2,3),(5,1,1)\}$ \\
\hline
\end{tabular}

Proof. A well-known fact is that the $n$-th Fibonacci number can be written as follows

$$
F_{n}=\frac{\alpha^{n}-\beta^{n}}{\alpha-\beta}, \text { where } \alpha=\frac{1+\sqrt{5}}{2} \text { and } \beta=\frac{1-\sqrt{5}}{2} .
$$

We also have that for all $n \geq 1$

$$
\alpha^{n-2} \leq F_{n} \leq \alpha^{n-1} .
$$

We note that in the Markoff case, $a=b=c$ and the equation is fully symmetric in $(x, y, z)$. This symmetry is no longer present in the case of the Rosenberg equation. In the proof we assume that $x \leq y \leq z$ hence we need to consider not only the equation $a x^{2}+b y^{2}+c z^{2}=d x y z$ but also all the permutations of $(a, b, c)$. We provide a bound for $i$ for general $(a, b, c, d)$ and we use it to get an upper bound for $k-j$. Based on inequalities from ([5]) we have

$$
\left.\frac{a F_{i}^{2}+b F_{j}^{2}}{F_{k}} \leq(a+b) \alpha^{j}, \quad\left|\frac{\beta^{k}}{\sqrt{5}}\right| \leq \frac{\alpha^{j}}{5}, \quad \mid \alpha^{i} \beta^{j}+\alpha^{j} \beta^{i}-\beta^{i+j}\right) \mid \leq 3 \alpha^{j} .
$$


Suppose $(x, y, z)=\left(F_{i}, F_{j}, F_{k}\right)$ for $i \leq j \leq k$ is a solution of

$$
a F_{i}^{2}+b F_{j}^{2}+c F_{k}^{2}=d F_{i} F_{j} F_{k} .
$$

We obtain that

$$
c \frac{\alpha^{k}}{\sqrt{5}}-d \frac{\alpha^{i+j}}{5}=-\frac{a F_{i}^{2}+b F_{j}^{2}}{F_{k}}+c \frac{\beta^{k}}{\sqrt{5}}-\frac{d}{5}\left(\alpha^{i} \beta^{j}+\alpha^{j} \beta^{i}-\beta^{i+j}\right) .
$$

Taking absolute values and using the inequalities at (2.1) we obtain:

$$
\left|c \frac{\alpha^{k}}{\sqrt{5}}-d \frac{\alpha^{i+j}}{5}\right| \leq \frac{\alpha^{j}}{5}(5 a+5 b+c+3 d)
$$

and dividing by $\frac{\alpha^{i+j}}{\sqrt{5}}$ :

$$
\left|c \alpha^{k-i-j}-\frac{d}{\sqrt{5}}\right| \leq \frac{5 a+5 b+c+3 d}{\sqrt{5} \alpha^{i}} .
$$

Now define $f(n)=\left|c \alpha^{n}-\frac{d}{\sqrt{5}}\right|$ and let $t_{0} \in \mathbb{Z}$ such that $f\left(t_{0}\right) \leq f(n)$ for any $n \in \mathbb{Z}$. Then

$$
\alpha^{i} \leq \frac{5 a+5 b+c+3 d}{\sqrt{5} f\left(t_{0}\right)} .
$$

For a given tuple $(a, b, c, d)$ equation (2.3) provides an upper bound for $i$, denote it by $\mathfrak{u} \mathfrak{b}(a, b, c, d)$. For a given $i$ equation (2.2) yields an upper bound for $k-j$. For the concrete equations we consider these bounds are as follows:

$$
\begin{aligned}
& \mathfrak{u} \mathfrak{b}(1,1,1,1)=9, \\
& \mathfrak{u} \mathfrak{b}(1,1,2,2)=8, \mathfrak{u} \mathfrak{b}(1,2,1,2)=\mathfrak{u} \mathfrak{b}(2,1,1,2)=9, \\
& \mathfrak{u} \mathfrak{b}(1,1,2,4)=\mathfrak{u} \mathfrak{b}(1,2,1,4)=\mathfrak{u b}(2,1,1,4)=8, \\
& \mathfrak{u b}(1,2,3,6)=\mathfrak{u} \mathfrak{b}(2,1,3,6)=8, \mathfrak{u} \mathfrak{b}(1,3,2,6)=\mathfrak{u} \mathfrak{b}(3,1,2,6)=7, \\
& \mathfrak{u b}(2,3,1,6)=\mathfrak{u} \mathfrak{b}(3,2,1,6)=11, \\
& \mathfrak{u} \mathfrak{b}(1,1,5,5)=7, \mathfrak{u} \mathfrak{b}(1,5,1,5)=\mathfrak{u} \mathfrak{b}(5,1,1,5)=8 .
\end{aligned}
$$

For each $(a, b, c, d)$ and any $i \leq \mathfrak{u b}(a, b, c, d)$ one needs to compute the (finitely many) possibilities for $m=k-j$. That is, fixing $(a, b, c, d), i$ and $m$ we study the equation

$$
a F_{i}^{2}+b F_{j}^{2}+c F_{j+m}^{2}-d F_{i} F_{j} F_{j+m}=0 .
$$

We note that the equation above only depends on $j$. To deal with the concrete cases we use the following arguments.

(I) We eliminate as many values of $i$ as possible by checking solvability of quadratic equations

$$
a F_{i}^{2}+b y^{2}+c z^{2}-F_{i} y z=0 .
$$

(II) For fixed $m$ we eliminate equations $a F_{i}^{2}+b F_{j}^{2}+c F_{j+m}^{2}-d F_{i} F_{j} F_{j+m}=0$ modulo $p$, where $p$ is a prime. 
(III) We consider the equation $a F_{i}^{2}+b F_{j}^{2}+c F_{j+m}^{2}=d F_{i} F_{j} F_{j+m}$ as a quadratic in $F_{j}$. Then its discriminant $d^{2} F_{i}^{2} F_{j+m}^{2}-4 b\left(a F_{i}^{2}+c F_{j+m}^{2}\right)$ must be a square. A fundamental identity for the Fibonacci and Lucas numbers (denoted by $L_{n}$, defined by $L_{0}=2, L_{1}=1$ and $L_{n}=L_{n-1}+L_{n-2}$ for $n \geq 2$ ) says that

$$
L_{n}^{2}=5 F_{n}^{2} \pm 4 .
$$

That is we have the system of equations

$$
\begin{aligned}
& Y_{1}^{2}=5 X^{2} \pm 4, \\
& Y_{2}^{2}=d^{2} F_{i}^{2} X^{2}-4 b\left(a F_{i}^{2}+c X^{2}\right),
\end{aligned}
$$

where $X=F_{j+m}$. Multiplying these equations together yields

$$
Y^{2}=\left(5 X^{2} \pm 4\right)\left(d^{2} F_{i}^{2} X^{2}-4 b\left(a F_{i}^{2}+c X^{2}\right)\right) .
$$

Therefore we reduce our problem to obtain integral points on the above quartic genus 1 curves. This will be realized using the Magma ([2]) function SIntegralLjunggrenPoints.

We implemented the above procedure in SageMath ([9]) and the code can be downloaded from the URL address http://shrek.unideb.hu/ tengely/ MarkoffSolver.sage. Detailed computations can be found at http:// shrek. unideb.hu/ tengely/Markoff-Rosenberger-Fibonacci.pdf.

2.1. The case with $d=1$. We have that $2 \leq i \leq 9$. In this range the Diophantine equation $F_{i}^{2}+y^{2}+z^{2}=F_{i} y z$ is solvable only for $i=4$. If $i=4$, then we have that $0 \leq k-j \leq 4$. The equation $9+F_{j}^{2}+F_{j+m}^{2}-3 F_{j} F_{j+m}=0$ has no solution modulo 3 for $m=1,2,3$, and it is not solvable modulo 11 for $m=4$. It remains to consider the case $m=0$. We have that $k=j$, therefore the equation is simply $9=F_{j}^{2}$. Hence, we get the solution $(x, y, z)=(3,3,3)$.

2.2. Cases with $d=2$. Consider the tuple $(a, b, c, d)=(1,1,2,2)$. The bound for $i$ is 8 , however only the quadratic equation related to $i=3$ is solvable in integers. If $i=3$, then $0 \leq k-j \leq 3$. We eliminate the cases $m=1,2$ modulo 7 and the case $m=3$ modulo 23 . If $k=j$, then we get that $4=F_{j}^{2}$. Hence, we obtain the solution $(x, y, z)=(2,2,2)$. There are 2 other subcases here, $(a, b, c, d)=(1,2,1,2)$ and $(2,1,1,2)$ having the same upper bound for $i$, namely 9 . In case of $(a, b, c, d)=(1,2,1,2)$ we can eliminate all values of $i$ except $i=3$ and 9 . If $i=3$ we have

$$
4+2 F_{j}^{2}+F_{j+m}^{2}-4 F_{j} F_{j+m}=0,
$$

where $0 \leq m \leq 5$. Congruence arguments eliminate the cases with $m \in$ $\{1,2,3,4,5\}$ as follows:

\begin{tabular}{|l||l|l|l|l|l|}
\hline$m$ & 1 & 2 & 3 & 4 & 5 \\
\hline $\bmod$ & 17 & 7 & 19 & 3 & 13 \\
\hline
\end{tabular}


The remaining value of $m$ is 0 , that yields the equation $4=F_{j}^{2}$, so we obtain the solution $(x, y, z)=(2,2,2)$. If $i=9$, then the corresponding equation is

$$
1156+2 F_{j}^{2}+F_{j+m}^{2}-68 F_{j} F_{j+m}=0
$$

where $0 \leq m \leq 9$. The following table contains the primes used to get a contradiction

\begin{tabular}{|l||l|l|l|l|l|l|l|l|l|l|}
\hline$m$ & 0 & 1 & 2 & 3 & 4 & 5 & 6 & 7 & 8 & 9 \\
\hline $\bmod$ & 3 & 7 & 11 & 19 & 11 & 5 & 11 & 7 & 3 & 29 \\
\hline
\end{tabular}

In case of $(a, b, c, d)=(2,1,1,2)$ we only need to handle $i=3$ for which we get that $0 \leq m \leq 5$. The equation is given by

$$
8+F_{j}^{2}+F_{j+m}^{2}-4 F_{j} F_{j+m}=0
$$

and we can eliminate all these (except $m=0$ ) as the table below shows

\begin{tabular}{|l||l|l|l|l|l|}
\hline$m$ & 1 & 2 & 3 & 4 & 5 \\
\hline $\bmod$ & 11 & 7 & 11 & 3 & 13 \\
\hline
\end{tabular}

If $m=0$, then we have $8=2 F_{j}^{2}$ and the only solution is $(x, y, z)=(2,2,2)$.

2.3. Cases with $d=4$. If $(a, b, c, d)=(1,1,2,4)$, then if follows that $i=2$ or 4 . If $(a, b, c, d)=(1,2,1,4)$, then we obtain that $i=2$ or 4 . The last tuple to consider here is $(a, b, c, d)=(2,1,1,4)$ and we get that $i=2$ or 5 . We need to handle the equations

$$
\begin{aligned}
1+F_{j}^{2}+2 F_{j+m}^{2}-4 F_{j} F_{j+m} & =0, \\
9+F_{j}^{2}+2 F_{j+m}^{2}-12 F_{j} F_{j+m} & =0, \\
1+2 F_{j}^{2}+F_{j+m}^{2}-4 F_{j} F_{j+m} & =0, \\
9+2 F_{j}^{2}+F_{j+m}^{2}-12 F_{j} F_{j+m} & =0, \\
2+F_{j}^{2}+F_{j+m}^{2}-4 F_{j} F_{j+m} & =0, \\
50+F_{j}^{2}+F_{j+m}^{2}-20 F_{j} F_{j+m} & =0 .
\end{aligned}
$$

We provide details in the case of the first equation, the other 5 can be solved in a similar way. We consider the equation as a quadratic in $F_{j}$ and follow the argument described in (III). It remains to solve the quartic Diophantine equations

$$
y^{2}=10 x^{4}-13 x^{2}+4, \quad y^{2}=10 x^{4}+3 x^{2}-4 .
$$

The integral solutions of these equations can be completely determined using the Magma ([2]) procedure SIntegralLjunggrenPoints. In the former case we get that $x \in\{0, \pm 1, \pm 5\}$. In case of the latter equation we have that $x \in\{ \pm 1\}$. It follows that $F_{j+m}=1$ or 5 and we get the solutions $(x, y, z)=$ $(1,1,1)$ and $(x, y, z)=(1,3,5)$. 
2.4. Cases with $d=5$. Here, we get the following possibilities for $i$ for the 3 tuples

\begin{tabular}{|c|c|}
\hline$(a, b, c, d)$ & $i$ \\
\hline$(1,1,5,5)$ & $\{2,3,4\}$ \\
\hline$(1,5,1,5)$ & $\{2,3,4\}$ \\
\hline$(5,1,1,5)$ & $\{2,3,5,7\}$ \\
\hline
\end{tabular}

Consider the tuple $(5,1,1,5)$. If $i=5$, then $0 \leq m \leq 7$ and if $i=7$, then $0 \leq m \leq 9$. All these cases can be eliminated using congruence arguments: if $i=5$, then we have

\begin{tabular}{|l||l|l|l|l|l|l|l|l|}
\hline$m$ & 0 & 1 & 2 & 3 & 4 & 5 & 6 & 7 \\
\hline $\bmod$ & 7 & 11 & 11 & 11 & 3 & 11 & 17 & 11 \\
\hline
\end{tabular}

and if $i=7$, then we obtain

\begin{tabular}{|l||l|l|l|l|l|l|l|l|l|l|}
\hline$m$ & 0 & 1 & 2 & 3 & 4 & 5 & 6 & 7 & 8 & 9 \\
\hline $\bmod$ & 3 & 11 & 13 & 29 & 11 & 19 & 11 & 29 & 3 & 11 \\
\hline
\end{tabular}

It remains to check the solutions for $i=2$ and 3 . The equations can be written as follows

$$
\begin{aligned}
5+F_{j}^{2}+F_{j+m}^{2}-5 F_{j} F_{j+m} & =0, \\
20+F_{j}^{2}+F_{j+m}^{2}-10 F_{j} F_{j+m} & =0 .
\end{aligned}
$$

As before we reduce the problem to genus 1 curves, we obtain the following 4 equations

$$
\begin{aligned}
& y^{2}=105 x^{4}-184 x^{2}+80 \\
& y^{2}=105 x^{4}-16 x^{2}-80, \\
& y^{2}=30 x^{4}-49 x^{2}+20, \\
& y^{2}=30 x^{4}-x^{2}-20 .
\end{aligned}
$$

The complete set of possible values for $F_{j}$ is given by $\{1,2,3,987\}$. We also know that $F_{i} \in\{1,2\}$, hence one can easily determine $F_{k}$. The solutions of the equation $x^{2}+y^{2}+5 z^{2}=5 x y z$ from these cases are given by $(x, y, z)=$ $(1,2,1),(2,1,1),(1,3,1),(3,1,1),(1,3,2)$ and $(3,1,2)$.

2.5. Cases with $d=6$. Let us consider the equation $x^{2}+2 y^{2}+3 z^{2}=6 x y z$. Here we can eliminate many quadratic equations. In the table below we collect the remaining cases. 


\begin{tabular}{|c|c|}
\hline$(a, b, c, d)$ & $i$ \\
\hline$(1,2,3,6)$ & $\{2,5\}$ \\
\hline$(2,1,3,6)$ & $\{2,3\}$ \\
\hline$(1,3,2,6)$ & $\{2,5\}$ \\
\hline$(3,1,2,6)$ & $\{2,4\}$ \\
\hline$(2,3,1,6)$ & $\{2,3\}$ \\
\hline$(3,2,1,6)$ & $\{2,4,11\}$ \\
\hline
\end{tabular}

We provide details in case of the tuple $(3,2,1,6)$ only, the remaining ones can be treated in a similar way. We have three values for $i$, these correspond to the equations

$$
\begin{aligned}
3+2 F_{j}^{2}+F_{j+m}^{2}-6 F_{j} F_{j+m} & =0, \\
27+2 F_{j}^{2}+F_{j+m}^{2}-18 F_{j} F_{j+m} & =0, \\
23763+2 F_{j}^{2}+F_{j+m}^{2}-534 F_{j} F_{j+m} & =0 .
\end{aligned}
$$

The last equation corresponds to $i=11$. Here, we do not expect any solution so we compute the possible values of $m$ and try to get a contradiction modulo some prime. It turns out that $0 \leq m \leq 13$ and all these cases can be handled using congruence arguments. We summarize the computation in the following table

\begin{tabular}{|l||l|l|l|l|l|l|l|l|l|l|l|l|l|l|}
\hline$m$ & 0 & 1 & 2 & 3 & 4 & 5 & 6 & 7 & 8 & 9 & 10 & 11 & 12 & 13 \\
\hline $\bmod$ & 5 & 17 & 19 & 7 & 13 & 5 & 17 & 13 & 7 & 17 & 13 & 13 & 17 & 29 \\
\hline
\end{tabular}

Solving the remaining two equations as described in (III) we get that we need to find the integral solutions of the Diophantine equations

$$
\begin{aligned}
& y^{2}=35 x^{4}-43 x^{2}+12 \\
& y^{2}=35 x^{4}+13 x^{2}-12 \\
& y^{2}=395 x^{4}-451 x^{2}+108 \\
& y^{2}=395 x^{4}+181 x^{2}-108 .
\end{aligned}
$$

We use the Magma function SIntegralLjunggrenPoints to determine the integral solutions and we get that $F_{j} \in\{1,2\}$. The tuple we consider is given by $(3,2,1)$ and the corresponding equation is $3 F_{i}^{2}+2 F_{j}^{2}+F_{k}^{2}=6 F_{i} F_{j} F_{k}$. Since $i=2$ or 4 we have $F_{i} \in\{1,3\}$. These possibilities yield the solutions $(x, y, z)=(1,1,1),(1,1,5),(1,2,1)$ and $(3,2,1)$.

\section{ACKNOWLEDGEMENTS.}

The author express his gratitude to the referees for careful reading of the manuscript and many valuable suggestions, which improve the quality of the paper. The research was supported by the National Scholarship Programme 
of the Slovak Republic. The author would like to thank the hospitality of the János Selye University during the project.

\section{REFERENCES}

[1] C. Baer and G. Rosenberger, The equation $a x^{2}+b y^{2}+c z^{2}=d x y z$ over quadratic imaginary fields, Results Math. 33 (1998), 30-39.

[2] W. Bosma, J. Cannon, and C. Playoust, The Magma algebra system. I. The user language, J. Symbolic Comput. 24 (1997), 235-265.

[3] E. González-Jiménez, Markoff-Rosenberger triples in geometric progression, Acta Math. Hungar. 142 (2014), 231-243.

[4] E. González-Jiménez and J. M. Tornero, Markoff-Rosenberger triples in arithmetic progression, J. Symbolic Comput., 53 (2013), 53-63.

[5] F. Luca and A. Srinivasan, Markov equation with Fibonacci components, Fibonacci Quart. 56 (2018), 126-129.

[6] A. Markoff, Sur les formes quadratiques binaires indéfinies, Math. Ann. 17 (1880), 379-399.

[7] G. Rosenberger, Über die diophantische Gleichung $a x^{2}+b y^{2}+c z^{2}=d x y z$, J. Reine Angew. Math. 305 (1979), 122-125.

[8] J. H. Silverman, The Markoff equation $X^{2}+Y^{2}+Z^{2}=a X Y Z$ over quadratic imaginary fields, J. Number Theory 35 (1990), 72-104.

[9] W. A. Stein et al., Sage Mathematics Software, version 8.5, The Sage Development Team, 2019. http://www. sagemath.org.

Sz. Tengely

Department of Mathematics

University of Debrecen

P.O.Box 12, 4010 Debrecen

Hungary

and

Department of Mathematics and Informatics

J. Selye University

Hradna ul. 21, 94501 Komarno

Slovakia

E-mail: tengely@science.unideb.hu

Received: 6.5.2019.

Revised: 10.9.2019. \& 23.11.2019. 